\title{
Global Stability and Hopf-bifurcation Analysis of Biological Systems using Delayed Extended Rosenzweig-MacArthur Model
}

\author{
Enobong E. Joshua ${ }^{1} \&$ Ekemini T. Akpan ${ }^{2}$ \\ ${ }^{1}$ Department of Mathematics \& Statistics, University of Uyo, Uyo \\ ${ }^{2}$ Department of Science Education, University of Uyo, Uyo \\ Correspondence: Ekemini T. Akpan, Department of Science Education, University of Uyo. P.M.B.1017, Uyo \\ AKS, Nigeria. Tel: 234-813-037-6772. E-mail: ekeminitakpan@uniuyo.edu.ng
}

Received: Dec. 10, 2017 Accepted: Dec. 19, 2017 Online Published: January 30, 2018

doi:10.5539/mas.v12n2p171 URL: https://doi.org/10.5539/mas.v12n2p171

\begin{abstract}
This paper investigates the global asymptotic stability of a Delayed Extended Rosenzweig-MacArthur Model via Lyapunov-Krasovskii functionals. Frequency sweeping technique ensures stability switches as the delay parameter increases and passes the critical bifurcating threshold.The model exhibits a local Hopf-bifurcation from asymptotically stable oscillatory behaviors to unstable strange chaotic behaviors dependent of the delay parameter values. Hyper-chaotic fluctuations were observed for large delay values far away from the critical delay margin. Numerical simulations of experimental data obtained via non-dimensionalization have shown the applications of theoretical results in ecological population dynamics.
\end{abstract}

Keywords: global asymptotic stability, Lyapunov-Krasovskii functionals, frequency sweeping technique, Hopfbifurcation, chaotic behaviors

\section{Introduction}

In Mathematical modeling of multiple interacting species, time-delays play important role on deterministic qualitative behaviors of dynamical systems. Recent advances in research on delay systems are found in (Gu, Kharitovov \& Chen, 2003; Lakshmanan \& Senthilkumar, 2010; Smith, 2010; Agarwal, O’Regan, \& Saker, 2014; Niculescu, $\& \mathrm{Gu}, 2004)$. In ecological population dynamical systems, changes in the environmental carrying capacities including maturation and gestation periods may not necessarily affect the entire population dynamics immediately. There are some time-past prior to the present state variables that affect the growth of the interacting species in the long run. These are classified as single or multiple delay parameters, state dependent delays, neutral-time delays, state dependent delays, and distributed delays. Also, the dynamical behaviors of such systems including global stability, instability, Hopf-bifurcations, strange chaotic attractor are induced by delayed parameters. (see, Feng \& Hongwei, 2012; Wang \& Li 2003; Xu \& Wu, 2014; Agrawal, Jana \& Upadhyay, 2014; Jatav \& Dhar, 2015). Thus, more realistic mathematical models in ecological population dynamics needs to incorporate a time lag or history between the moment in which an action takes place and the moment its effect is actually observed in the dynamical system. There are recent studies in dynamical behaviors of a 3-D Rosenzweig-MacArthur Model (1963) such as uniform boundedness, local and global asymptotic stabilities, local Hopf-bifurcation/limit cycles, persistence and permanence, global attractors, existence and uniqueness of positive periodic solution (see, Feng, Freeze, Lu \& Rocco, 2014; Joshua, Akpan, Adebimpe \& Madubueze, 2016; Joshua \& Akpan 2016; Joshua \& Akpan, 2017). However, to the best of the authors' knowledge little emphasis has been placed on the effects of time-delay parameter on the model. Thus, in this paper, the effect of time-delay bifurcation parameters on the dynamical behaviors of the model will be studied. 


\section{Model Formulation and Invariance State Space}

Consider the ERMM studied in (Freng, Freeze, Lu, and Rocco, 2014) given as:

$$
\left\{\begin{array}{l}
\frac{d x_{1}}{d t_{1}}=r x_{1}-\frac{r x_{1}^{2}}{K}-a_{2} \frac{x_{1}}{b_{1}+x_{1}} x_{2}-a_{3} \frac{x_{1}}{b_{1}+x_{1}} x_{3} \\
\frac{x_{2}}{d t_{1}}=c_{2} a_{2} \frac{x_{2}}{b_{1}+x_{1}} x_{3}-d_{2} x_{2}-a_{3} \frac{x_{2}}{b_{2}+x_{2}} x_{3} \\
\frac{b_{1}}{d t_{1}}=c_{3} a_{3} \frac{x_{2}}{b_{2}+x_{2}} x_{3}-d_{3} x_{3}+c_{3} a_{3} \frac{x_{1}}{b_{1}+x_{1}} x_{3}
\end{array}\right.
$$

where $x_{1}\left(t_{1}\right), x_{2}\left(t_{2}\right)$, and $x_{3}\left(t_{3}\right)$ are the population densities of the interacting species; $r, K, a_{2}, a_{3}, b_{1}, b_{2}, c_{2}, c_{3}, d_{2}$ and $d_{3}$ are positive ecological parameters. Introduce a single discrete-time delay parameter to the system (1) and obtain a topologically equivalence system (2) via non-dimensionalization of the state variables;

$$
\left\{\begin{array}{l}
\frac{d x}{d t}=\alpha x-\frac{\alpha x^{2}}{\kappa}-\frac{\eta x y}{1+x}-\frac{x z}{1+x} \\
\frac{d y}{d t}=\frac{\varepsilon x(t-\tau) y}{1+x(t-\tau)}-\xi y-\frac{\sigma y z}{1+y} \\
\frac{d z}{d t}=\frac{\beta y(t-\tau) z}{1+y(t-\tau)}-\mu z+\frac{\beta x(t-\tau) z}{1+x(t-\tau)}
\end{array}\right.
$$

where $x(t)=\frac{x_{1}\left(t_{1}\right)}{b_{1}}, y(t)=\frac{x_{2}\left(t_{1}\right)}{b_{2}}, z(t)=\frac{x_{3}\left(t_{1}\right)}{b_{1}}, \alpha=\frac{r}{a_{3}}, \kappa=\frac{K}{b_{1}}, \eta=\frac{a_{2} b_{2}}{a_{3} b_{1}}, \varepsilon=\frac{c_{2} a_{2}}{a_{3}}, \xi=\frac{d_{2}}{a_{3}}, \sigma=$ $\frac{b_{1}}{b_{2}}, \mu=\frac{d_{3}}{a_{3}}, c_{3}=\beta, t=a_{3} t_{1}$ and subject to initial conditions; $x(t)=\phi_{1}(t) \geq 0, \phi_{1}(0)>0 ; y(t)=\phi_{2}(t) \geq$ $0, \phi_{2}(0)>0 ; z(t)=\phi_{3}(t) \geq 0, \phi_{3}(0)>0, \forall t \in[-\tau, 0]$, where $\left(\phi_{1}(t), \phi_{2}(t), \phi_{3}(t)\right) \in C\left([-\tau, 0], \mathbb{R}_{+}^{3}\right)$ is a Banach space of continuous functions mapping on the interval $[-\tau, 0]$ into $\mathbb{R}_{+}^{3}=(x(t), y(t), z(t) \mid x(t)>0, y(t)>0, z(t)>$ $0)$. The fundamental theory of functional differential equation guarantees existence and uniqueness of solution $(x(t), y(t), z(t))$ of system (2) and forward invariance in $[0,+\infty) \forall t>0$ see (Gopalsamy, 1992; Arino, Hbid \& Dads, 2006; Michiels \& Niculescu, 2014).

\section{Existence of Coexisting Equilibrium Point}

The system (2) can be represented as $\dot{X}(t)=\mathbf{F}(X(t), X(t-\tau))$ where $X(t)=(x(t), y(t), z(t)) \in \mathbb{R}_{+}^{3} \tau \geq 0$ is a discrete delay-in-time parameter and $\mathbf{F}$ a vector valued continuous function. Generally, the behaviors of the dynamical system (2) at steady-state are independent of the delay parameter and satisfies $\mathbf{F}\left(X(t)=X(t-\tau)=X^{*}=0\right)$ and $X^{*}=\left(x^{*}, y^{*}, z^{*}\right) \in \mathbb{R}_{+}^{3}$ being any fixed point of system (2). Observe that, the model depicts an ecological population dynamical system. Thus, the interest will be focused on the behavior of the coexisting equilibrium point $E^{*}\left(x^{*}, y^{*}, z^{*}\right)$ satisfying the following conditions;

$$
\left\{\begin{array}{l}
y^{*}=\frac{\mu\left(1+x^{*}\right)-\beta x^{*}}{(\beta-\mu)\left(1+x^{*}\right)+\beta x^{*}} ; z^{*}=\frac{\beta\left(\varepsilon x^{*}-\xi\left(1+x^{*}\right)\right)}{\sigma\left((\beta-\mu)\left(1+x^{*}\right)+\beta x^{*}\right)} ; \text { and } \frac{\xi}{\varepsilon-\xi}<x^{*}<\frac{\mu}{\beta-\mu} \\
P\left(x^{*}\right)=x^{* 3}+A_{1} x^{* 2}+A_{2} x^{*}+A_{3}=0 ; \text { where } A_{1}=\frac{2 \alpha \beta \kappa \sigma-\alpha \sigma \kappa \mu-3 \alpha \sigma \beta+2 \alpha \sigma \mu}{\alpha \sigma \mu-2 \alpha \sigma \beta} \\
A_{2}=\frac{3 \alpha \beta \kappa \sigma-2 \alpha \sigma \kappa \mu+\kappa \sigma \eta \beta-\kappa \sigma \eta \mu-\alpha \sigma \beta+\alpha \sigma \mu+\kappa \beta \xi-\beta \kappa \varepsilon}{\alpha \sigma \mu-2 \alpha \sigma \beta} ; A_{3}=\frac{\alpha \sigma \beta \kappa-\alpha \sigma \kappa \mu+\kappa \beta \xi}{\alpha \sigma \mu-2 \alpha \sigma \beta}
\end{array}\right.
$$

By applying Descartes' Rule of Sign (Wiggins, 2003), system (2) has a unique positive equilibrium point $E^{*}\left(x^{*}, y^{*}, z^{*}\right)$ if there exists a unique positive real root $x^{*}$ of the polynomial in (3), whence $A_{1}>0, A_{2}>0, A_{3}>0$ and

$$
\left\{\begin{array}{l}
x^{*}=\sqrt[3]{-\frac{q}{2}+\sqrt{\left(\frac{q}{2}\right)^{2}+\left(\frac{p}{3}\right)^{3}}}+\sqrt[3]{-\frac{q}{2}-\sqrt{\left(\frac{q}{2}\right)^{2}+\left(\frac{p}{3}\right)^{3}}}-\frac{A_{1}}{3} \\
p=A_{2}-\frac{A_{1}^{2}}{3}, \text { and } q=\frac{1}{27}\left(2 A_{1}^{3}-9 A_{1} A_{2}+27 A_{3}\right)
\end{array}\right.
$$

\section{Global Asymptotic Stability}

Definition 1: The time-delay dynamical system (2) is stable independent of delay for all nonnegative delay parameters, otherwise the system's stability is dependent on delay. 
Lemma 1( Cerone \& Dragomir, 2011): If $p, q \geq 1$ is defined with conjugacy $\frac{1}{p}+\frac{1}{q}=1$, then $\frac{1}{p} x^{p}+\frac{1}{q} y^{q} \geq$ $x y ; \quad \forall x, y \geq 0$

Proposition 1: If the dynamical system (2) is locally asymptotically stable, then the unique fixed point $E^{*}\left(x^{*}, y^{*}, z^{*}\right)$ is globally asymptotically stable independent of delay parameter (in the sense of Lyapunov), given a LyapunovKrasovskii functional $V(x, y, z): C\left([-\tau, 0], \mathbb{R}_{+}^{3}\right) \rightarrow \mathbb{R}_{+}$satisfying

$$
\dot{V}(x, y, z) \leq-X M X^{T}
$$

where $X=\left(\left|x(t)-x^{*}\right|,\left|y(t)-y^{*}\right|,\left|z(t)-z^{*}\right|,\left|x(t-\tau)-x^{*}\right|,\left|y(t-\tau)-y^{*}\right|\right)$ and $M$ being a real positive definite and symmetric diagonal matrix.

Proof Consider a candidate Lyapunov-Krasovskii functional given as

$$
\begin{aligned}
V(x, y, z) & \stackrel{\text { def }}{=} V_{1}(x, y, z)+V_{2}(x, y, z)+V_{3}(x, y, z) \\
& \equiv\left(x-x^{*}-x^{*} \log _{e}\left(\frac{x}{x^{*}}\right)+\delta_{1} \int_{t-\tau}^{t}\left(x(s)-x^{*}\right)^{2} d s\right)+y-y^{*}-y^{*} \log _{e}\left(\frac{y}{y^{*}}\right)+ \\
& \delta_{2} \int_{t-\tau}^{t}\left(y(s)-y^{*}\right)^{2} d s+\left(z-z^{*}-z^{*} \log _{e}\left(\frac{z}{z^{*}}\right)\right)
\end{aligned}
$$

Now using lemma 1, the directional derivative of the Lyapunov-Krasovskii functional $V(x, y, z)$ along the solution trajectories of system (2) yields;

$$
\left\{\begin{aligned}
& \dot{V}_{1}(x, y, z)=\left(\frac{\eta y^{*}+z^{*}}{1+x^{*}}-\frac{2 \alpha+\kappa \eta+\kappa-2 \kappa \delta_{1}}{2 \kappa}\right)\left(x-x^{*}\right)^{2}-\frac{\eta}{2}\left(y-y^{*}\right)^{2}-\frac{1}{2}\left(z-z^{*}\right)^{2}- \\
& \delta_{1}\left(x(t-\tau)-x^{*}\right)^{2} \\
& \dot{V}_{2}(x, y, z)=\left(\frac{\varepsilon}{2\left(1+x^{*}\right)}+\frac{\sigma y^{*}+2 \delta_{2}\left(1+y^{*}\right)-\sigma\left(1+y^{*}\right)}{2\left(1+y^{*}\right)}\right)\left(y-y^{*}\right)^{2}-\frac{\sigma}{2}\left(z-z^{*}\right)^{2}+ \\
& \frac{\varepsilon}{2\left(1+x^{*}\right)}\left(x(t-\tau)-x^{*}\right)^{2}-\delta_{2}\left(y(t-\tau)-y^{*}\right)^{2} \\
& \dot{V}_{3}(x, y, z)=\left(\frac{\beta\left(x^{*}+y^{*}+2\right)}{2\left(1+x^{*}\right)\left(1+y^{*}\right)}\right)\left(z-z^{*}\right)^{2}+\left(\frac{\beta}{2\left(1+x^{*}\right)}\right)\left(x(t-\tau)-x^{*}\right)^{2}+ \\
&\left(\frac{\beta}{2\left(1+y^{*}\right)}\right)\left(y(t-\tau)-y^{*}\right)^{2}
\end{aligned}\right.
$$

The quadratic bilinear form of equations (6) yields;

$$
\left\{\begin{aligned}
\dot{V}(x, y, z) \stackrel{\text { def }}{=} & \dot{V}_{1}(x, y, z)+\dot{V}_{2}(x, y, z)+\dot{V}_{3}(x, y, z) \\
\leq & -\left(\psi_{1}\left(x(t)-x^{*}\right)^{2}+\psi_{2}\left(y(t)-y^{*}\right)^{2}+\psi_{3}\left(z(t)-z^{*}\right)^{2}+\psi_{4}\left(x(t-\tau)-x^{*}\right)^{2}+\right. \\
& \left.\psi_{5}\left(y(t-\tau)-y^{*}\right)^{2}\right) \\
\dot{V}(x, y, z) \leq & -X M X^{T}
\end{aligned}\right.
$$

Choose $\delta_{1}=\delta_{2}=\beta, X=\left(\left|x(t)-x^{*}\right|,\left|y(t)-y^{*}\right|,\left|z(t)-z^{*}\right|,\left|x(t-\tau)-x^{*}\right|,\left|y(t-\tau)-y^{*}\right|\right)$ and $M=\operatorname{diag}\left(\psi_{1}, \psi_{2}, \psi_{3}, \psi_{4}, \psi_{5}\right)$ being a positive symmetric matrix defined as;

$$
M=\left(\begin{array}{ccccc}
\psi_{1} & 0 & 0 & 0 & 0 \\
0 & \psi_{2} & 0 & 0 & 0 \\
0 & 0 & \psi_{3} & 0 & 0 \\
0 & 0 & 0 & \psi_{4} & 0 \\
0 & 0 & 0 & 0 & \psi_{5}
\end{array}\right)
$$




$$
\left\{\begin{array}{l}
\psi_{1}=\frac{\left(2 \alpha+\kappa \eta+\kappa-2 \kappa \delta_{1}\right)\left(1+x^{*}\right)-2 \kappa\left(\eta y^{*}+z^{*}\right)}{2 \kappa\left(1+x^{*}\right)} \\
\psi_{2}=\frac{\eta\left(1+x^{*}\right)\left(1+y^{*}\right)-\varepsilon\left(1+y^{*}\right)-\left(1+x^{*}\right)\left[\sigma y^{*}+2 \delta_{2}\left(1+y^{*}\right)-\sigma\left(1+y^{*}\right)\right]}{2\left(1+x^{*}\right)\left(1+y^{*}\right)} \\
\psi_{3}=\frac{(\sigma+1)\left(1+x^{*}\right)\left(1+y^{*}\right)-\beta\left(x^{*}+y^{*}+2\right)}{2\left(1+x^{*}\right)\left(1+y^{*}\right)} \\
\psi_{4}=\frac{2 \delta_{1}\left(1+x^{*}\right)-(\varepsilon+\beta)}{2\left(1+x^{*}\right)} \\
\psi_{5}=\frac{2 \delta_{2}\left(1+y^{*}\right)-\beta}{2\left(1+y^{*}\right)}
\end{array}\right.
$$

Observe that the Lyapunov-Krasovskii functional satisfies

$$
\left\{\begin{array}{l}
V(x, y, z)=0 \text { at }\left(x^{*}, y^{*}, z^{*}\right) \in \mathbb{R}_{+}^{3} \\
V(x, y, z)>0 ; \quad \forall(x, y, z) /\left(x^{*}, y^{*}, z^{*}\right) \in \mathbb{R}_{+}^{3}
\end{array}\right.
$$

and $E\left(x^{*}, y^{*}, z^{*}\right)$ is a global minimum of $V(x, y, z)$. Thus $V(x, y, z)$ is a strictly positive definite functional. Similarly, it is clear from (7) that the symmetric matrix $M$ satisfies the Sylvester's criterion for which all the upper-left leading principal minors of $M$ are positive, and $\dot{V}(x, y, z)<0$. Using Lyapunov-LaSalle invariance principle (LaSalle, 1968), the fixed point $E\left(x^{*}, y^{*}, z^{*}\right)$ is global attractor trapped in the largest invariance subset of $\mathbb{R}_{+}^{3}$. Hence every trajectory of system (2) is attracted to coexisting equilibrium point $E\left(x^{*}, y^{*}, z^{*}\right)$;

\section{Local Hopf-Bifurcation Analysis via Frequency Sweeping Technique}

In this section, frequency-domain technique is employed to investigates the distribution of roots of characteristic quasi-polynomial of system (2) in the upper-right half complex plane, critical bifurcating delay margin $\left(\tau^{*}>0\right)$, zero-crossing frequency number $\left(\omega^{*}>0\right)$ from stable region to unstable region, and transversality condition. However, since only finitely many unstable roots may be in the open right half-plane, there are only finite number of zero crossings. For details on frequency-sweeping technique see (Li, Niculescu \& Cela, 2015)

Lemma 2: [Ruan \& Wei, 2003] Consider the transcendental equation

$$
\begin{aligned}
p\left(\lambda, e^{-\lambda \tau_{1}}, \ldots, e^{-\lambda \tau_{m}}\right)= & \lambda^{n}+p_{1}^{(0)} \lambda^{n-1}+\ldots+p_{n-1}^{(0)} \lambda+p_{n}^{(0)}+\left[p_{1}^{(1)} \lambda^{n-1}+\ldots+p_{n-1}^{(1)} \lambda+p_{n}^{(1)}\right] e^{-\lambda \tau_{1}}+\ldots \\
& +\left[p_{1}^{(m)} \lambda^{n-1}+\ldots+p_{n-1}^{(m)} \lambda+p_{n}^{(m)}\right] e^{-\lambda \tau_{m}}
\end{aligned}
$$

as $\left(\tau_{1}, \tau_{2}, \ldots, \tau_{m}\right)$ vary, the sum of orders of the zeros of $p\left(\lambda, e^{-\lambda \tau_{1}}, \ldots, e^{-\lambda \tau_{m}}\right)$ in the open right half plane can change, and only a zero appears on or crosses the imaginary axis.

Remark 1: This means that the number of characteristic roots with positive real parts can change only if there exist a pair of imaginary roots.

Lemma 3 [ Gu, Kharitonov \& Ghen, 2003] The time-delay dynamical system (2), defined as;

$$
\dot{X}(t)=A_{0} X(t)+A_{1} X(t-\tau)
$$

(under appropriate initial conditions, where $X(t) \in \mathbb{R}_{+}^{3}$ is the system steady state at time $t, A_{0}, A_{1} \in \mathbb{R}^{3 \times 3}$ are real matrices, and $\tau \in[0,+\infty)$ is the delay parameter) is asymptotically stable independent of delay if and only if the following conditions are satisfied;

$$
\left\{\begin{array}{l}
A_{0} \text { is asymptotically stable } \\
A_{0}+A_{1} \text { is asymptotically stable } \\
\rho\left(\left(i \omega I-A_{0}\right)^{-1} A_{1}\right)<1 \quad \forall \omega>0
\end{array}\right.
$$

where $\rho($.$) denotes a spectral radius.$

For proof of lemma 2 see details in (Gu, Kharitonov \& Ghen, 2003) . 
Remark 2: When the system is not stable independent of delay, it remains possible to extend the above lemmas to compute the critical delay margin( i.e the first stability interval, including $\tau=0$ ) which characterized the delay interval governing asymptotic stability of the system.

Proposition 2: Assume that lemmas 2 and 3 hold, then the positive coexisting equilibrium point $E\left(x^{*}, y^{*}, z^{*}\right)$ is asymptotically stable dependent of delay when $\tau \in\left[0, \tau^{*}\right)$, unstable for $\tau \in\left[\tau^{*}, \infty\right)$ and system (2) undergoes a local sub-critical Hopf-bifurcation at the positive equilibrium point $E\left(x^{*}, y^{*}, z^{*}\right)$ for $\tau \in\left(\tau^{*}-\epsilon, \tau^{*}+\epsilon\right)$ satisfying the transversality condition; $\mathfrak{R} e\left(\frac{d \lambda}{d \tau}\right)_{\tau=\tau^{*}, \lambda=i \omega^{*}} \neq 0$

Proof: The linear perturbation of system (2) near the steady state $E\left(x^{*}, y^{*}, z^{*}\right)$ say, $P(t)=x-x^{*}, Q(t)=$ $y-y^{*}, R(t)=z-z^{*}, P_{\tau}=x(t-\tau)-x^{*}, Q_{\tau}=y(t-\tau)-y^{*} ; R_{\tau}=z(t-\tau)-z^{*}$ using Taylor's expansion and neglecting higher order terms yields;

$$
\left(\begin{array}{c}
\dot{P}(t) \\
\dot{Q}(t) \\
\dot{R}(t)
\end{array}\right)=\left(\begin{array}{ccc}
\alpha_{11} & \alpha_{12} & \alpha_{13} \\
0 & \alpha_{22} & \alpha_{23} \\
0 & 0 & \alpha_{33}
\end{array}\right)\left(\begin{array}{l}
x(t) \\
y(t) \\
z(t)
\end{array}\right)+\left(\begin{array}{ccc}
0 & 0 & 0 \\
\beta_{21} & 0 & 0 \\
\beta_{31} & \beta_{32} & 0
\end{array}\right)\left(\begin{array}{l}
x(t-\tau) \\
y(t-\tau) \\
z(t-\tau)
\end{array}\right)
$$

where

$$
\left\{\begin{array}{l}
A_{0}=\left(\begin{array}{ccc}
\alpha_{11} & \alpha_{12} & \alpha_{13} \\
0 & \alpha_{22} & \alpha_{23} \\
0 & 0 & \alpha_{33}
\end{array}\right), A_{1}=\left(\begin{array}{ccc}
0 & 0 & 0 \\
\beta_{21} & 0 & 0 \\
\beta_{31} & \beta_{32} & 0
\end{array}\right) ; \alpha_{12}=-\frac{\eta x^{*}}{\left(1+x^{*}\right)} \\
\alpha_{11}=\frac{\left(\alpha \kappa-2 \alpha x^{*}\right)\left(1+x^{*}\right)^{2}-\kappa\left(\eta y^{*}+z^{*}\right)}{\kappa\left(1+x^{*}\right)^{2}} ; \alpha_{13}=-\frac{x^{*}}{1+x^{*}} ; \alpha_{23}=-\frac{\sigma y^{*}}{1+y^{*}} \\
\alpha_{22}=\frac{\left(1+y^{*}\right)^{2}\left[\varepsilon x^{*}-\xi\left(1+x^{*}\right)\right]}{\left(1+x^{*}\right)\left(1+y^{*}\right)} ; \alpha_{33}=\frac{\left(1+x^{*}\right)\left[\beta y^{*}-\mu\left(1+y^{*}\right)\right]+\beta x^{*}\left(1+y^{*}\right)}{\left(1+x^{*}\right)\left(1+y^{*}\right)} \\
\beta_{21}=\frac{\varepsilon y^{*}}{\left(1+x^{*}\right)^{2}} ; \beta_{31}=\frac{\beta z^{*}}{\left(1+x^{*}\right)^{2}} ; \beta_{32}=\frac{\beta z^{*}}{\left(1+y^{*}\right)^{2}}
\end{array}\right.
$$

Define the characteristic quasi-polynomial of system (12) in terms of the ecological parameters as;

$$
\begin{cases}p\left(\lambda, e^{-\lambda \tau}\right) & =\lambda^{3}+\zeta_{1} \lambda+\zeta_{2} \lambda+\zeta_{3}+\left(\zeta_{4}+\zeta_{5} \lambda\right) e^{-\lambda \tau}+\zeta_{6} e^{-2 \lambda \tau}=0 \\ \zeta_{1} & =-\left(\alpha_{11}+\alpha_{22}+\alpha_{33}\right) ; \zeta_{2}=\alpha_{11} \alpha_{22}+\alpha_{22} \alpha_{33}+\alpha_{11} \alpha_{33} ; \zeta_{3}=-\alpha_{11} \alpha_{22} \alpha_{33} \\ \zeta_{4} & =\alpha_{11} \alpha_{23} \beta_{32}+\alpha_{12} \alpha_{33} \beta_{21}-\alpha_{12} \alpha_{23} \beta_{31}+\alpha_{13} \alpha_{22} \beta_{31} \\ \zeta_{5} & =-\left(\alpha_{23} \beta_{32}+\alpha_{12} \beta_{21}+\alpha_{31} \beta_{31}\right) ; \zeta_{6}=-\alpha_{13} \beta_{21} \beta_{32}\end{cases}
$$

Let $\lambda=\alpha^{*} \pm i \omega^{*} \quad \forall\left(\alpha^{*}, \omega^{*}\right) \in \mathbb{R}$ be a pair of spectrum associated with the characteristic quasi-polynomial (11), if the critical delay margin $\tau^{*}=0$, then lemma 2 and Routh-Hurwitz criterion, see (Wiggins, 2003) guarantee the asymptotic stability region of system (2) for which $\alpha^{*}<0$. Similarly, assume that lemma 1 holds, and the critical delay margin $\tau^{*}>0$, then there is a stability switch when the value of $\alpha^{*}$ crosses the imaginary axis at $\lambda= \pm i \omega^{*}$. Thus, the quasi-polynomial (13) satisfies the conjugate symmetric condition; $p\left(\lambda, e^{\lambda \tau}\right)_{\lambda= \pm i \omega^{*}}=0$, leading to expression of the critical bifurcating delay margin $\tau=\tau^{*}$ as follows;

$$
\tau^{*}=\frac{1}{\omega^{*}} \arccos \left(\frac{\zeta_{5} \omega^{*}\left(\omega^{*^{3}}-\zeta_{2} \omega^{*}\right)+\zeta_{4}\left(\zeta_{1} \omega^{*^{2}}+\zeta_{6}-\zeta_{3}\right)}{\left(\zeta_{3}-\zeta_{1} \omega^{*^{2}}-\zeta_{6}\right)\left(\zeta_{3}-\zeta_{1} \omega^{*^{2}}+\zeta_{6}\right)+\left(\omega^{*^{3}}-\zeta_{2} \omega^{*}\right)^{2}}\right)+\frac{2 n \pi}{\omega^{*}} ; n=0, \pm 1, \ldots
$$

and its positive zero-crossing frequencies satisfy the equation;

$$
\left\{\begin{aligned}
\phi\left(\omega^{*}\right)= & \omega^{12}+\alpha_{0} \omega^{10}+\alpha_{1} \omega^{8}+\alpha_{2} \omega^{6}+\alpha_{3} \omega^{4}+\alpha_{4} \omega^{2}+\alpha_{5} \\
\alpha_{0}= & 2 \zeta_{1}^{2}-4 \zeta_{2} ; \alpha_{1}=\zeta_{1}^{2}-4 \zeta_{1}^{2} \zeta_{2}-4 \zeta_{1} \zeta_{3}+6 \zeta_{2}^{2}-\zeta_{5}^{2} \\
\alpha_{2}= & 2 \zeta_{1}^{2} \zeta_{2}^{2}-4 \zeta_{1}^{3} \zeta_{3}-\zeta_{1}^{2} \zeta_{5}^{2}+8 \zeta_{1} \zeta_{2} \zeta_{3}-4 \zeta_{2}^{3}+2 \zeta_{2} \zeta_{5}^{2}+2 \zeta_{3}^{2}-\zeta_{4}^{2}-2 \zeta_{6}^{2} \\
\alpha_{3}= & 6 \zeta_{1}^{2} \zeta_{3}^{2}-\zeta_{1}^{2} \zeta_{4}^{2}-2 \zeta_{1}^{2} \zeta_{6}^{2}+2 \zeta_{1} \zeta_{5}^{2} \zeta_{6}+2 \zeta_{1} \zeta_{5}^{2} \zeta_{3}-4 \zeta_{1} \zeta_{2}^{2} \zeta_{3}-4 \zeta_{2} \zeta_{3}^{2}+\zeta_{2}^{4}-\zeta_{2}^{2} \zeta_{5}^{2}+ \\
& 2 \zeta_{2} \zeta_{4}^{2}+4 \zeta_{2} \zeta_{6}^{2}-\zeta_{4} \zeta_{5} \zeta_{6} \\
= & 2 \zeta_{1} \zeta_{4}^{2} \zeta_{3}+4 \zeta_{1} \zeta_{3} \zeta_{6}^{2}-4 \zeta_{1} \zeta_{3}^{3}-2 \zeta_{1} \zeta_{4}^{2} \zeta_{6}+2 \zeta_{2}^{2} \zeta_{3}^{2}-\zeta_{3}^{2} \zeta_{5}^{2}-2 \zeta_{3} \zeta_{5}^{2} \zeta_{6}-\zeta_{2}^{2} \zeta_{4}^{2}-2 \zeta_{2}^{2} \zeta_{6}^{2}+ \\
& 4 \zeta_{2} \zeta_{4} \zeta_{5} \zeta_{6}-\zeta_{5}^{2} \zeta_{6}^{2} \\
= & \left(\zeta_{3}-\zeta_{6}\right)^{2}\left(\zeta_{4}+\zeta_{3}+\zeta_{6}\right)\left(\zeta_{3}-\zeta_{4}+\zeta_{6}\right) \\
\alpha_{5} \quad &
\end{aligned}\right.
$$


By applying Descartes' Rules of sign, the polynomial in (15) has a unique positive zero-crossing frequency leading to a stability switch at the coexisting equilibrium point, and dependent of the computed critical bifurcating delay margin in equation (14). Furthermore, to ensure a local sub-critical Hopf-bifurcation for the critical pair $\left(\tau^{*}, \omega^{*}\right)$,using equation (13) the transversality condition yields;

$$
\operatorname{sgn} \mathfrak{R} e\left(\frac{d \lambda}{d \tau}\right)_{\tau=\tau^{*}, \lambda=i \omega^{*}}=\left\{\begin{array}{l}
\operatorname{sgn} \mathfrak{R}\left(\frac{3 \lambda^{2}+2 \zeta_{1} \lambda+\zeta_{2}+\zeta_{5} e^{-\lambda \tau}}{\lambda\left(\zeta_{4}+\zeta_{5} \lambda\right) e^{-\lambda \tau}+2 \lambda \zeta_{6} e^{-2 \lambda \tau}}-\frac{\tau}{\lambda}\right)_{\tau=\tau^{*}, \lambda=i \omega^{*}} \\
\operatorname{sgn} \mathfrak{R}\left(\frac{3 \lambda^{2}+2 \zeta_{1} \lambda+\zeta_{2}+\zeta_{5} e^{-\lambda \tau}}{\lambda\left(\zeta_{4}+\zeta_{5} \lambda\right) e^{-\lambda \tau}+2 \lambda \zeta_{6} e^{-2 \lambda \tau}}\right)_{\tau=\tau^{*}, \lambda=i \omega^{*}} \\
\left(\frac{Q R-P S}{\omega^{*}\left(R^{2}+S^{2}\right)}\right) \neq 0 \\
\text { where; } \\
P=\zeta_{2}-3 \omega^{*}+\zeta_{5} \cos \omega^{*} \tau^{*} ; Q=2 \zeta_{1} \omega^{*}-\zeta_{5} \sin \omega^{*} \tau^{*} \\
R=\zeta_{4} \cos \omega^{*} \tau^{*}+\zeta_{5} \omega^{*} \sin \omega^{*} \tau^{*}+2 \zeta_{6} \cos 2 \omega^{*} \tau^{*} ; \\
S=\zeta_{5} \omega^{*} \cos \omega^{*} \tau^{*}-\zeta_{4} \sin \omega^{*} \tau^{*}-2 \zeta_{6} \sin 2 \omega^{*} \tau^{*}
\end{array}\right.
$$

Therefore, the transversality condition holds and Hopf-bifurcation occurs at the critical pair $\left(\tau^{*}, \omega^{*}\right)$. Using cluster treatment of the characteristic quasi-polynomial in (13), see (Niculescu, \& Gu, 2004). The Root Tendency(RT) destabilizes the coexisting equilibrium point with increase in the number of positive roots which leads to a subcritical Hopf-bifurcation.

Remark 3: The determination of critical pair $\left(\tau^{*}, \omega^{*}\right)$ is the defining feature of frequency sweeping technique. The results show that, if the gestation delay parameter $\tau$ is in the cluster $\left[0, \tau^{*}\right)$ the model is stable at the equilibrium point $E\left(x^{*}, y^{*}, z^{*}\right)$. Also, a Hopf-bifurcation occurs at $\tau=\tau^{*}$, and the model degenerates to an unstable oscillatory behavior for $\tau \in\left(\tau^{*},+\infty\right]$.

\section{Numerical Simulations}

Table 1. Ecological parameters/variables of model simulations **

\begin{tabular}{llll}
\hline$* *$ & Descriptions & values* & $*$ (Feng et al 2014) \\
\hline$x(t)$ & prey population density & variable & variable \\
$y(t)$ & predator population density & variable & variable \\
$z(t)$ & super-predator population density & variable & variable \\
$\alpha$ & intrinsic growth rate of the prey & 0.7688 & 0.6750 \\
$\kappa$ & environmental carrying capacity of the prey & 2.0064 & 0.9430 \\
$\eta$ & maximum predation rate of the predator & 0.1673 & 0.1580 \\
$\varepsilon$ & predator biomass conversion efficiency & 0.1249 & 0.6940 \\
$\xi$ & independent death rate of the predator species & 0.0410 & 0.0360 \\
$\sigma$ & maximum predation rate of the super-predator & 1.0755 & 0.8780 \\
$\beta$ & super-predator biomass conversion efficiency & 0.3655 & 0.5430 \\
$\mu$ & independent death rate of the super-predator & 0.3804 & 0.3340 \\
$\tau$ & gestation delay parameter during predation effects & $\tau \in[0,+\infty)$ & $* * * * *$ \\
$\delta_{1}$ & free parameter & 0.3655 & $* * * * *$ \\
$\delta_{2}$ & free parameter & 0.3655 & $* * * * *$ \\
$x(0)$ & initial condition of prey species & 1.0678 & 1.5000 \\
$y(0)$ & initial condition of predator species & 1.3730 & 0.5000 \\
$z(0)$ & initial condition of super-predator species & 0.6385 & 0.3000 \\
$x^{*}$ & coexisting equilibrium point of preys & 1.8519 & 0.9210 \\
$y^{*}$ & coexisting equilibrium point of predators & 0.6431 & 0.0171 \\
$z^{*}$ & coexisting equilibrium point of super-predators & 0.5307 & 0.0191 \\
\hline
\end{tabular}

In this section, a set of experimental data was generated via non-dimensionalization to verify the theoretical results (see table 1). All ecological parameters including the fixed point and initial conditions remain unchanged, and gestation delay parameter will be varied to control the dynamics of the system. 
In figure (1) the dynamical system (2) exhibit periodic oscillatory behaviors independent of any delay parameter. The system is locally asymptotically stable at the coexisting equilibrium point for all ecological parameters in table 1 and the Jacobian matrix computed as;

$$
\left(\begin{array}{ccc}
-0.7288496405 & -0.1086366146 & -0.6493521495 \\
0.00987680473 & -0.1712850651 & -0.4209641462 \\
0.02384821978 & 0.07183889708 & -5.197 * 10^{-7}
\end{array}\right)
$$

yields the negative spectra $(-0.09751206712 \pm 0.1569067171 i,-0.7051110911 i)$. Observe that theorem 1 was satisfied because the symmetric matrix $M$ is positive definite with positive spectra computed using ecological parameters of table 1 ,

$$
M=\left(\begin{array}{ccccc}
0.2506759932 & 0 & 0 & 0 & 0 \\
0 & 0.01599436954 & 0 & 0 & 0 \\
0 & 0 & 0.8624497402 & 0 & 0 \\
0 & 0 & 0 & 0.2795211469 & 0 \\
0 & 0 & 0 & 0 & 0.2542806350
\end{array}\right)
$$

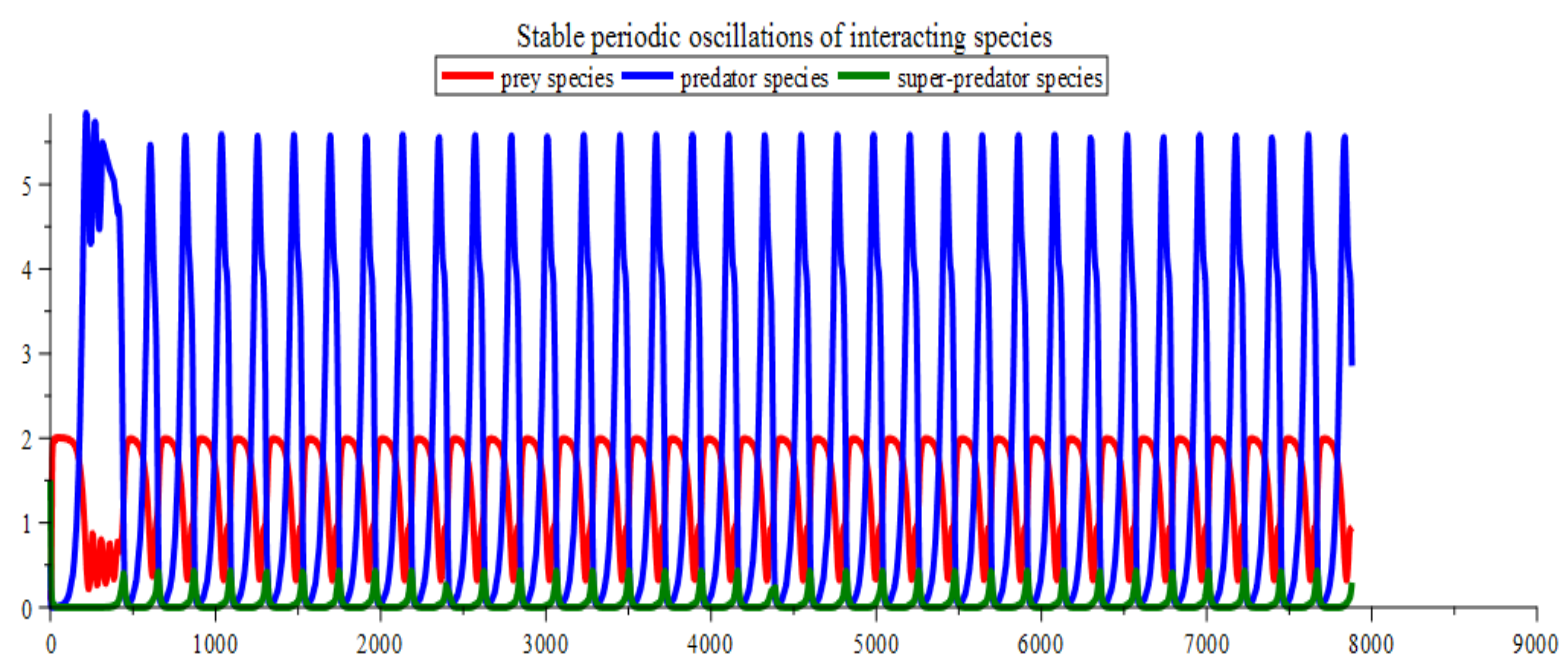

Figure 1. Simulation of global asymptotic stable behaviors of dynamical system (2) at coexisting equilibrium point for delay parameter $\tau=0$ or $\tau=+\infty$.

Thus, the system is globally asymptotically stable in the sense of Lyapunov, and every trajectory converges to the coexisting equilibrium point. Biologically, the populations of the interacting species will persist for a long time without occurrence of extinction scenarios amongst species.

In figure (2), the system is asymptotically stable for a small perturbation near the coexisting equilibrium point. Observe that lemma 2 was satisfied, real matrices $A_{0}$ and $A_{0}+A_{1}$ have negative spectra $(-0.7288496028,-3.007036669 *$ $\left.10^{-3},-5.221 * 10^{-7}\right)$ and $\left(-0.705471615508848,-2.67244380405758 * 10^{-2}+0.174059720093838 i,-2.67244380405758 *\right.$ $\left.10^{-2}-0.174059720093838 i\right)$ respectively.

Similarly, the maximum spectral radius yields $\rho()=.0.52516<1, \omega^{*}=0.2477>0$ for a delay parameter value $\left(\tau \approx 0.02029<\tau^{*} \approx 0.3799\right.$.) Thus, a small delay gestation parameter is harmless to the dynamical system, and the system remains stable for $\tau \in[0,0.3799)$; otherwise it bifurcates to unstable behaviors for $\tau \in[0.3799,+\infty)$. 


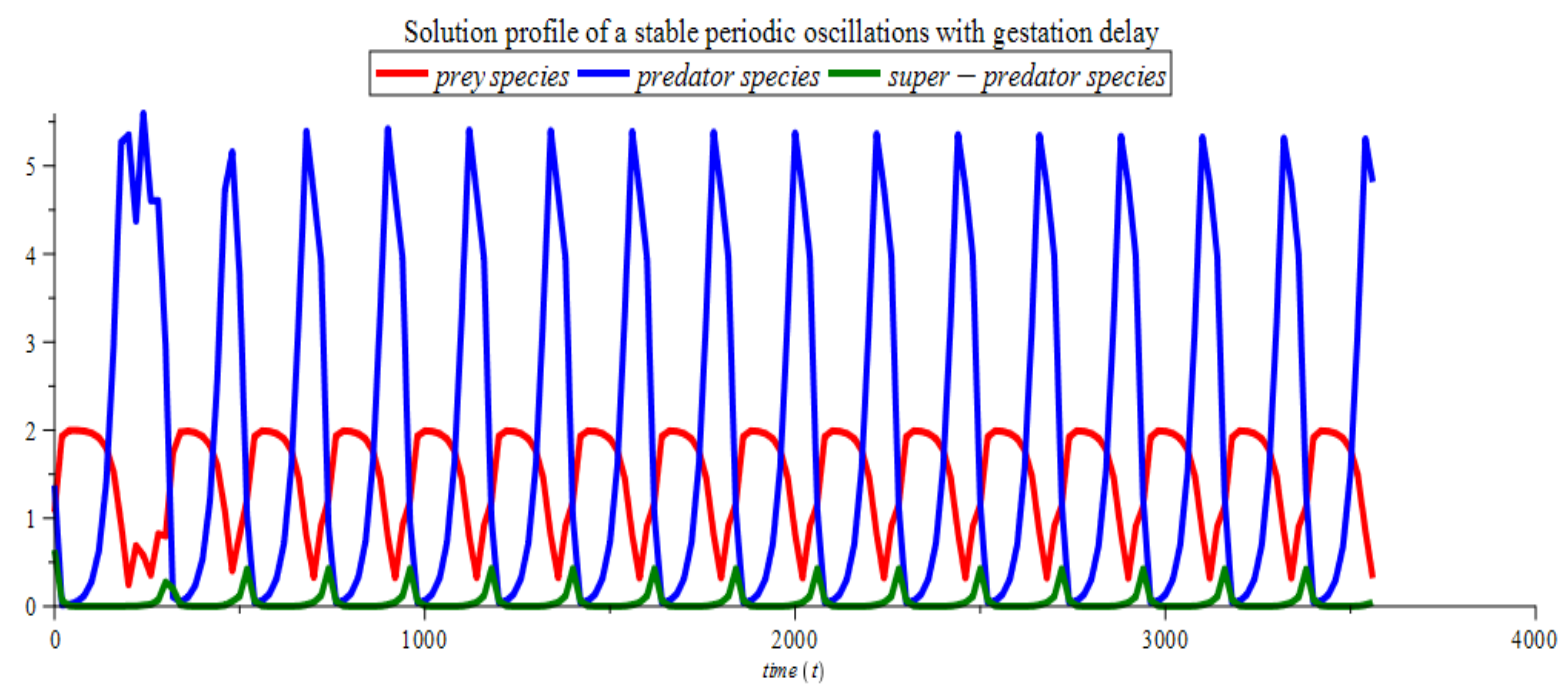

Figure 2. Simulation of stable periodic oscillatory behaviors of system (2) at coexisting equilibrium point for delay parameter $\tau \approx 0.02029<\tau^{*} \approx 0.3799$.

In figure (3) a route to strange chaotic behaviors was observed as the the gestation delay parameter increases and passes a critical bifurcating threshold value $\left(\tau \approx 3.0589>\tau^{*} \approx 0.3799\right)$. This leads to unstable behaviors of the dynamical system (2) near the coexisting equilibrium point (see, table 1). The simulation given in figure (4) has shown a hyper-chaotic regime as the delay parameter increases far away the critical delay value, $\tau \approx 3.0609>$ $\tau^{*} \approx 0.3799$

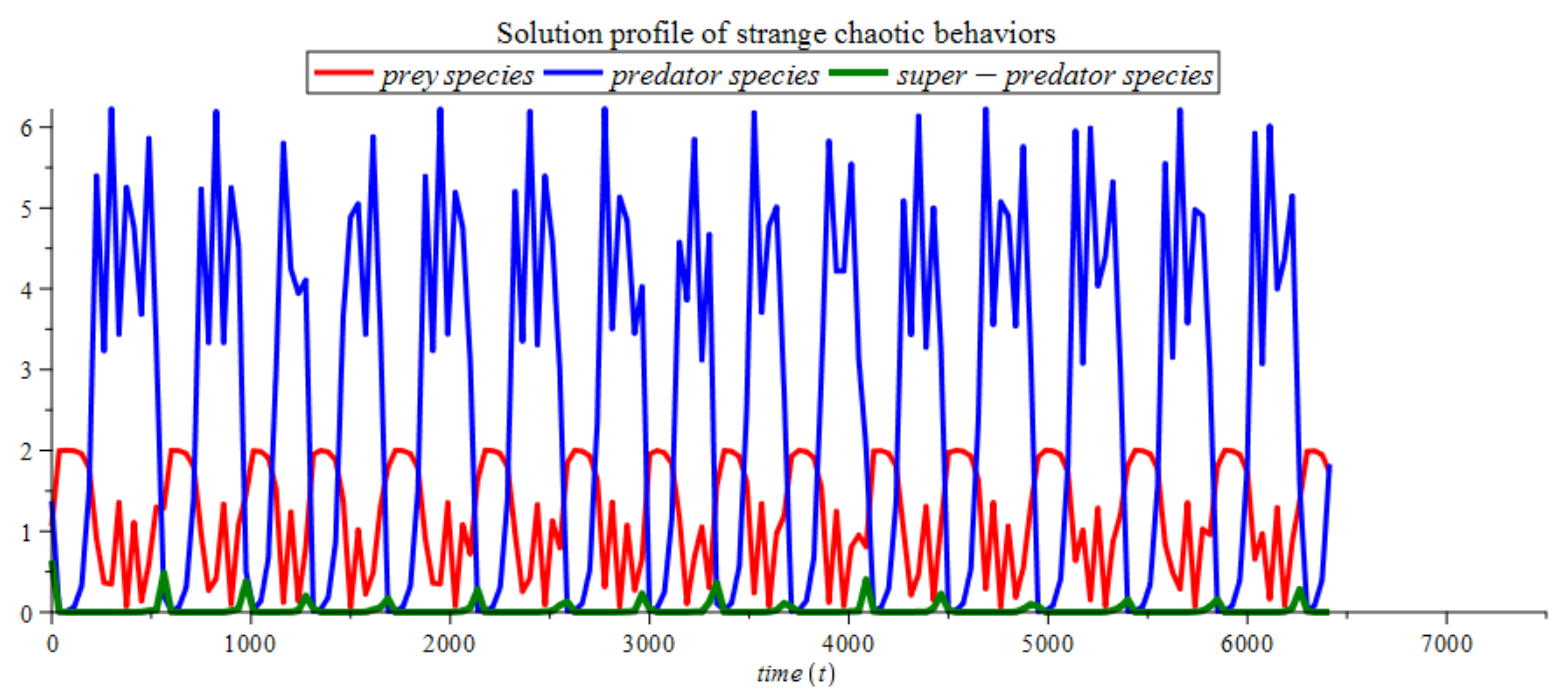

Figure 3. Simulation of strange chaotic behaviors of dynamical system (2) near the coexisting equilibrium point for delay parameter value $\tau \approx 3.0589>\tau^{*} \approx 0.3799$ 


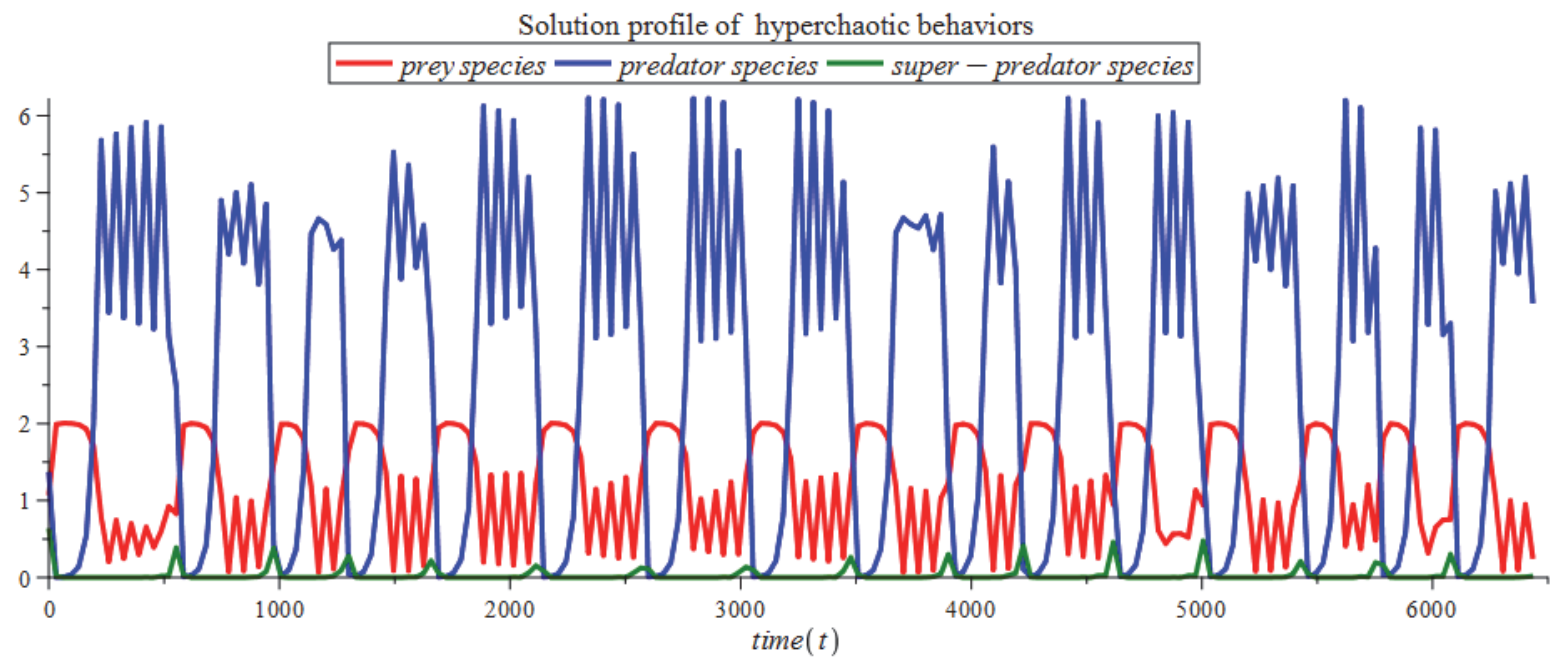

Figure 4. Simulation of strange hyper-chaotic behaviors of dynamical system (2) near the coexisting equilibrium point for delay parameter value $\tau \approx 3.060938632>\tau * \approx 0.3799$

\section{Conclusion}

In this paper, we established the qualitative dynamical behaviors of a biological system formulated via Delayed Extended Rosenzweig-MacArthur Model. The su_cient conditions of existence of coexisting equilibrium point was obtained. The model exhibits global asymptotic stable behaviors in the sense of Lyapunov. It is shown that gestation time delay parameter plays important role in controlling the dynamical behaviors of the system. When delay parameter increases beyond the minimum critical bifurcating value the system degenerates from a local Hopf-bifurcation near the coexisting equilibrium point to strange chaotic/hyper-chaotic dynamics. Numerical sim-Hopf-bifurcation near the coexisting equilibrium point to strange chaotic/hyper-chaotic dynamics. Numerical simulations have shown the feasibility of theoretical results and biological implications of the model. It predicts a stabilized system for small gestation delay parameter values, and un-stabilized system for large delay parameter values. Thus, the small delay values are harmless to the dynamical system and preserve species extinction scenarios, while large delay values require the use of Lyapunov's exponent and power spectrum analysis to predict further dynamics of the system.

\section{Acknowledgment}

This work was funded by the Tertiary Education Trust Fund(TetFund) for Institution-Based Research(TetFund/IBR/2016) intervention in Nigeria.

\section{References}

Agarwal, R. P., V. L. O'Regan, \& Saker, S. H. (2014). Oscillation and stability of delays models in biology. New York: Springer-Verlag. https://doi.org/10.1007/978-3-319-06557-1

Arino, O., M. L. Hbid., \& Dads, E. A. (2006). Delay di_erential equations and applications, Nether lands: Springer Inc.

Cerone, P., \& Dragomir, S. S. (2011). Mathematical inequalities. New York: Taylors and Francis Group, LLC.

Feng, L., \& Hongwei, L. (2012). Hopf bifurcation of a predator prey model with time delay and t stage structure for the prey. Mathematical and Computer Modelling, 55, 672-679. Elsevier.

Gopalsamy, K. (1992). Stability and oscillation in delay di_erential equations of population dynamics, Netherlands: Kluwer Academic Publishers.

Gu, K., Kharitonov, V. L., \& Chen, J. (2003). Stability of time-delay systems. New York: Springer Science+Business Media, LLC.

Jana, D., Agrawal, R., \& Upadhyay, R. K. (2014). Top-predator interference and gestation delay as determinant of the dynamics of a realistic model food chain. Chaos, Solitons, and Fractals, 69, 50-63. Elsevier.

Jatav, K. S., \& Dhar, J. (2015). Global behavior and Hopf bifurcation of a stage structure prey predator model 
with maturation delay for prey and gestation delay predator. Journal of Biological Sciences, 23(1), 57-77. World Scientific Publishing Company. https://doi.org/10.1142/S0218339015500047

Joshua, E. E., \& Akpan, E. T. (2016). Persistence and global dynamics of extended Rosenzweig MacArthur model. Mathematical Theory and Modelling, 6(8), 85-99.

Joshua, E. E., Akpan, E. T., \& Madubueze, C. E. (2016). Hopf bifurcation limit cycles of an extended Rosenzweig-MacArthur model. Journal of Mathematics Research, 8(3), 22-32. https://doi.org/10.5539/jmr.v8n3p22

Joshua, E. E., Akpan, E. T., Adebimpe, O., \& Madubueze, C. E. (2017) Existence and uniqueness of positive periodicsolutions of an extended Rosenzweig-MacArthur model via Brouwers' topological degree. British Journal of Mathematics and Computer Science, 20(4) 1-10. https://doi.org/10.9734/BJMCS/2017/31342

Lakshmanan, M., \& Senthilkumar, D. V. (2010). Dynamics of nonlinear time-delay systems. Heidelberg: Springer-Verlag.

LaSelle, J. (1968). Stability theory for ordinary di_erential equations. Journal of Di_erential Equations, 4, 57-65.

Li, X. G., Niculescu, I. S., \& Cela, A. (2015). Analytic curve frequency-sweeping stability test for system with commensurate delays. Springer-Verlag, New York. https://doi.org/10.1007/978-3-319-15717-7

Michiels, W., \& Niculescu, S. (2014). Stability, Control, and Computation for Time-Delay Systems: Eigenvalue-Based Approach. USA: Society of Industrial and Applied Mathematics(SIAM).

Niculescu, S. I., \& Gu, K. (2004). Advances in time-delay systems. Springer-Verlag, Berlin. https://doi.org/10.1007/978-3-642-18484-6

Rosenzweig, M. L., \& MacArthur, R. H. (1963). Graphical representation and stability conditions of predator-preyinteractions. American Naturalist, 97, 209-223.

Ruan, S., \& Wei, J. (2003). On the zeros of transcendental functions with applications to stability of delay di_erential equations with two delays. Dynamics of Continuous Discrete Implusive Systems Series A: Math. Anal, 10(1), 863-874.

Smith, H. (2011). An introduction to delay di_erential equations with applications in the life sciences. New York: Springer Science + Business Media. https://doi.org/10.1007/978-4419-7646-8

Wiggins, S. (2003) Introduction to applied nonlinear dynamical systems and chaos. New York: Springer- Verlag, Inc.

$\mathrm{Xu}, \mathrm{C} .$, \& Wu, Y. (2014) Bifurcation and control of chaos in a chemical system. Appl. Maths. Modelling. http://dx.doi/101016/j.apm.2014.10.030

\section{Copyrights}

Copyright for this article is retained by the author(s), with first publication rights granted to the journal.

This is an open-access article distributed under the terms and conditions of the Creative Commons Attribution license (http://creativecommons.org/licenses/by/3.0/). 